\title{
Physiognomie und Schicksal? Oder wie der altmesopotamische Mensch mit einem durch ein physiognomisches Omen angekündigtes Unheil umgegangen sein mag
}

Barbara BöcK CSIC Madrid

\section{EINLEITUNG}

Die Physiognomik, die «Kenntnis der Körperlichkeit», mag vielleicht als Urform der Menschenkenntnis und -deutung gelten. Blicken wir auf unsere westlich-moderne Geistesgeschichte, so kommt der Name Johann Caspar LAVATERs (1741-1801) in den Sinn, der durch sein vierbändiges Werk Physiognomische Fragmente zur Beförderung der Menschenkenntnis und Menschenliebe (Leipzig - Winterthur 1775-1778), die Physiognomik als Lehrsystem populär machte. Zweck seiner Physiognomik ist neben der Beurteilung anderer auch die Selbsterziehung: Der Mensch solle durch das Studium der eigenen Physiognomie überprüfen, wie weit sie von eigennützigen bzw. edleren Zügen geprägt ist, und gegebenenfalls den eigenen Lebenswandel ändern.

Bereits in der Antike erfreute sich die Disziplin der Physiognomie großer Aufmerksamkeit, wenn wir an die Physiognomika des Pseudo-Aristoteles, die Charaktere Theophrasts oder an die in 
arabischer Übersetzung erhaltenen Schriften des Polemon von Laodikeia denken. Letzterer ein Rhethor zur Zeit Hadrians, der seine physiognomische Menschenkenntnis dazu nutzte, politische Gegner zu diffamieren. Von einer bewusst eingesetzten Physiognomie schließlich zeugt das Werk Quintilians über den Rhetorischen Gebrauch von Mimik und Gesten.

Hauptquelle der keilschriftlichen Physiognomik oder Morphoskopie ist das akkadische Omenhandbuch Alamdimmû, 'Gestalt'. Das Werk, das in mehrere Abschnitte eingeteilt rund 27 Tafeln aufweist, in denen die Physiognomie von Mann und Frau gedeutet werden, welche Auslegungen zur Erscheinung von Hautveränderungen bieten und die ferner auch die Interpretation menschlichen Verhaltens einschließen, wird in seinem vollen Umfang erst in der Bibliothek Assurbanipals in Ninive greifbar ${ }^{1}$.

Doch anders als etwa die antiken Werke mit ihrem eher philosophisch-naturwissenschaftlich geprägten Ansatz wurde die altmesopotamische Physiognomik als Kommunikationsmittel der göttlichen mit der irdischen Welt aufgefasst. So geht beispielsweise dem Kapitel über allgemeine Verhaltensnormen namens Kataduggh, 'Ausspruch', von F. R. KRAUS seinerzeit auch mit 'Sittenkanon' ${ }^{2}$ bezeichnet, eine kurze Einleitung voraus, in welcher als Herkunft die göttliche Sphäre und als Zweck dieses Abschnittes eine Art «Lebenshilfe» genannt werden: «Als die großen Götter die Seele der Menschheit zur Ausübung der Macht bestimmt und sie mit ihrem 'Ausspruch' zu ihrem ständigen Geleit versehen hatten» ${ }^{3}$. Nach einem spekulativen Text galt das Omenhandbuch Alamdimmû sowie die Kataduggĥu-Verhaltensomina sogar als Werk des Gottes Ea ${ }^{4}$.

Die Omenform der keilschriftlichen Physiognomik weist das Handbuch in den Bereich der Religion, was sich nicht zuletzt auch daran zeigt, dass der āsipu, der 'Beschwörer', sich für diese Diszi-

\footnotetext{
1 S. mein Die babylonisch-assyrische Morphoskopie (= BaMo), AfO Bh. 27 (Wien 2000), Kap. II S. 9-18.

2 «Ein Sittenkanon in Omenform», ZA 43 (1936) S. 77-113. S. für die Bearbeitung unter Berücksichtigung neuer Textfragmente, BaMo, S. 130-147, Tf. 6-8.

3 Enūma ilānu rabiūtu ša amēèùti zaqũqša ana illilūiti šaknū u kataduggâsa ana reteddīsa ukinnū; für die Textvertreter s. BaMo, S. 130.

4 K 2248 Vs. 2-4; publiziert von W. G. LAMBERT, «A Catalogue of Texts and
} Authors», JCS 16 (1962) S. 64. 
plin zuständig zeichnete 5 . Die physiognomischen Omina wurden, ähnlich anderen Omensammlungen auch, zum Ende des 2. Jt.s v.Chr. hin systematisch gesammelt und in einem verwissenschaftlichenden Prozess zu Handbüchern gestaltet. Sicherlich sind einige der Beobachtungen Einsichten, die Volkes Weisheit entsprangen, doch müssen wir davon ausgehen, dass der überwiegende Teil der Omina artifziell hinzugefügt wurde - eine Entwicklung, die ich auf die Sorge eines horror vacui culturalis zurückgeführt hatte ${ }^{6}$. Einblick in diesen verwissenschaftlichenden Prozess bietet eine Passage aus einem Katalog mit den Tafelanfängen der prognostischdiagnostischen und morphoskopischen Omenkompendia, Sakikk $\hat{u}$ 'Symptome' und Alamdimmû, die von der Kompilierung und Kanonisierung der beiden Korpora durch den Weisen Esagil-kīn-apli zeugt. Der Abschnitt ist mehrmals übersetzt und diskutiert worden ${ }^{7}$; von Interesse hier ist eine Aussage, die für den Zusammenhang menschlicher Körperlichkeit und menschlichem Los spricht: «Das Handbuch Alamdimmû über Physiognomie und Gestalt birgt in sich das Schicksal der Menschheit, welche Ea und Asaluhi ihr bestimmten.»

\section{SCHUTZ VOR EINEM UNHEILVOLLEN SCHICKSAL}

Eines der bekanntesten Abwehrmechanismen von durch Omina angezeigter Übel ist das sog. Namburbi-Löseritual ${ }^{8}$. Der Ritualablauf verläuft je nach Art und Form des Omenanzeigers verschie-

\footnotetext{
${ }^{5}$ Alamdimmû wird als eines der Werke, die zum Curriculum eines Beschwörers gehören, genannt; s. KAR 44 Z. 6. Vgl. für die Bearbeitung und Duplikate des Textes M. J. GELLER, «Incipits and Rubrics», in Wisdom, Gods and Literature: Studies in Honour of W. G. Lambert, eds. A. R. GEORGE und I. L. FINKEL (Winona Lake Indiana 2000) S. 225-258. Nach der Tafel STT I 37 Rs. 24-25 konnte der mašmaššuBeschwörer nur unter Schwierigkeiten die Omenserie hersagen; vgl. die Bearbeitung von W. G. LAMBERT, Babylonian Wisdom Literature (Oxford 1960) S. 211-212.

${ }^{6}$ «Babylonische Divination und Magie als Ausdruck der Denkstrukturen des altmesopotamischen Menschen», in Babylon: Focus mesopotamischer Geschichte, Wiege früher Gelehrsamkeit, Mythos in der Moderne (II. Internationales Colloquium der Deutschen Orient-Gesellschaft e.V.), ed. J. RENGER (Berlin 1999) S. 424-425.

7 S. die Bearbeitung des Textes durch I. L. FINKEL, "Adad-apla-iddina, Esagilkīn-apli, and the Series SA.GIG», in A Scientific Humanist: Studies in Memory of Abraham Sachs, ed. E. LEICHTY et al. (Philadelphia 1988) S. 143-159; vgl. ferner B. BöcK, BaMo, S. 55 und N. HEEßEL, Babylonisch-assyrische Diagnostik (= AOAT 43) (Münster 2000) S. 104

8 S. S. M. MAUL, Zukunftsbewältigung (= BaFo 18) (Mainz 1994).
} 
den und mehr oder weniger kompliziert: der Omenanzeiger (etwa Tier, Pflanze, meteorologische Erscheinung, usf.) musste eingefangen oder in Form eines Modells substituiert, ein Gerichtsverfahren vor Šamaš angestrengt, die Götter mithilfe zahlreicher Gebete und Opfer gnädig gestimmt, der betroffenene Mensch kultisch gereinigt und schließlich wieder in die Gesellschaft eingegliedert werden. Während derartige apotropäische Rituale für Omina der Handbücher Šumma ālu, Enūma anu enlil, Šumma izbu, Iqqur ̄̄puš und auch für durch Opferschau vorausgesagte Übel bezeugt sind, fehlen bislang Löserituale, die sich auf das Handbuch Alamdimm $\hat{u}$ beziehen könnten ${ }^{9}$. Man mag hier vielleicht den Textbefund verantwortlich machen -nicht alle der mehr als 140 Tafeln des Namburbi-Ritualwerkes Assurbanipals sind auf uns gekommen ${ }^{10}$ - oder den Umstand, dass ein Teil der physiognomischen Omendeutungen sich auf den Charakter und die Eigenheiten eines Menschen beziehen. So fragt sich E. REINER diesbezüglich, ob man nicht aufgrund der Absenz von Namburbi-Ritualen für das Alamdimmî-Handbuch möglicherweise zwischen zwei Typen von divinatorischen Texten unterscheiden muss: Ein Typus von Omen dient hauptsächlich der Prophezeiung zukünftiger Ereignisse (z.B. die Omenkompendia Šumma ālu, Enūma anu enlil, Šumma izbu, Iqqur $\bar{\imath} p u s ̌$ ), während die Deutungen des anderen Typus, repräsentiert durch die prognostisch-diagnostische Omenserie Sakikk $\hat{u}$ und unser Alamdimm $\hat{u}$, diagnostischer Natur sind ${ }^{11}$. Etwas später kommt sie zu dem Schluss, dass «evidently these attributes, for example, being pusillanimous, or honest, or affectionate (...), or scratching one's nose when speaking (...), even if they portended misfortune [they] were not thougt to be susceptible of change through a ritual $(\ldots){ }^{12}$.

E. LEICHTY argumentierte seinerzeit, ob nicht die Auffälligkeit einer ominösen Erscheinung als Kriterium für die Durchführung von Ritualen zur Abwendung des durch den Omenanzeiger angekündigten Unheils diente. So führt er an, dass «minor anomalies such as birthmarks must have been handled in a completely different manner» ${ }^{13}$.

\footnotetext{
${ }^{9}$ S. S. M. MAUL, Zukunftsbewältigung, S. 12 Anm. 100.

${ }^{10}$ S. S. M. MAUL, Zukunftsbewältigung, S. 216-217.

${ }^{11}$ Astral Magic in Babylonia (= TAPS 85/4) (Philadelphia 1995) S. 84-85.

12 E. Reiner, Astral Magic in Babylonia, S. 85.

${ }_{13}$ The Omen Series Summa izbu (= TCS IV) (Locust Valley - N.Y. 1970) S. 12.
} 
In der Tat scheinen oftmals einfache, magische Handlungen genügt zu haben. A. L. OPPENHEIM und F. KÖCHER machten Ende der 60er Jahre auf ein kleines Fragment aufmerksam, welches zu dem Typ der Šumma ălu-Omina gehört. Die kurzen Anleitungen in diesem Text nach einer ungünstigen Apodose «are quite unique in Mesopotamian omen literature» ${ }^{14}$. Einige der Zeilen dieses Textes seien zur Illustrierung zitiert:

Funck 3

Z. 26

šumma harimtu ana pānī̌su lā kišitti șummirāti tulêša ilappitma pašir

«Wenn eine <unreine> Prostituierte vor ihm steht, Nicht-Erreichen der Wünsche; er soll ihre Brust berühren und es ist gelöst.»

Z. 28

šumma ina sūqi haṣbattu ana pāñ̄̌š zaqpat lä kišitti șummirāti ina šēpišu ša šumēli ikabbassima pašir

«Wenn in der Straße eine Tonscherbe seitlich stehend vor ihm ist, Nicht-Erreichen der Wünsche; er soll mit dem linken Fuß auf sie treten und es ist gelöst.»

Z. 31

[šumma karpat pāhari] ana pānī̌š lā kišitti șummirāti kurgarrâ inațalma pašir

«[Wenn ein Töpferge]fäß vor ihm ist, Nicht-Erreichen der Wünsche; er soll einen kurgarrû-Priester ansehen und es ist gelöst.»

Zwar bot das Gebet keinen Schutz vor zukünftigem Unglück, doch diente es neben dem Opfer als Mittel per se, die Götter zu besänftigen und dazu zu stimmen, ein schlimmes Los zu ändern. So ist es nicht verwunderlich, wenn wir die in den Omenapodosen angekündigten Facetten von Unglück in den Klageteilen von Gebete und Hymnen finden, wie etwa (a) in einem Gebet an Ištar

14 «The Old-Babylonian Omen Text VAT 7525», AfO 18 (1958) S. 71. 
und (b) in der Marduk-Hymne Ludlul bēl nēmeqi, «Preisen will ich den Herrn der Weisheit!».

a) Gebet an Ištar ${ }^{15}$

Z. $89 u l$ kunnā išdā $[\check{s} u]$, «[seine Füße] stehen nicht sicher» ${ }^{16}$

Z. 155 dūssu etrr $[e t]$, «seine Manneskraft ist fortgenom[en]»

Parallel dazu sind die Aposen:

TBP Nr. 22 Z. 34 (= BaMo, S. 266)

išdāšu ul kunnā «seine Füße stehen nicht sicher»

šumma umșātu Z. 1 (= BaMo, s. 184)

dūssu itenetțir, «seine Manneskraft wird fortgenommen»

(b) Ludlul bēl nēmeqi ${ }^{17}$

Tafel II Z. 5

usalli ${ }^{\mathrm{d} i s ̌ t a r} \bar{\imath}$ ul ušaqqā rēš $\bar{s}$ a,

«Ich betete zu meiner Göttin, aber sie kümmert sich nicht um mich (wörtl.: erhebt ihren Kopf nicht)»

Parallel dazu ist die Apodose einer altbabylonischen Tafel über Körpermale:

Šumma umșātum (TBP 62) Z. 17 (= BaMo, S. 302)

ilšu rēššš ušaqqā, «Sein Gott wird seinen Kopf nicht erheben»

Übereinstimmungen zwischen ungünstiger Omenapodose und Klageteil bzw. als in das positiv gekehrte Gegenteil im Bittteil findet sich bei den rituell eingebetteten Suila-Gebeten. Auf diesen Umstand ist bislang nicht hingewiesen worden, zeigt er doch die besondere Nähe zwischen der Omenliteratur und dem rituellen Gebet. Ob diese Beobachtung allein mit der wachsenden Standardisierung literarischer Texte allgemein ${ }^{18}$, die eine zunehmende

${ }^{15}$ Veröffentlicht und bearbeitet von W. G. LAMBERT, «Three Literary Prayers of the Babylonians», AfO 19 (1959-1960) S. 50-55.

${ }^{16} \mathrm{Vgl}$. zu dieser Wendung die Ausführungen von E. CASSIN, «Le droit et le tordu II», Le semblable et le différent (Paris 1987) S. 73-76.

${ }^{17} \mathrm{~S}$. für die Bearbeitung W. G. LAMBERT, Babylonian Wisdom Literature (Oxford 1960) S. 21-62 und S. 283-302.

${ }^{18}$ S. A. L. Oppenheim, Ancient Mesopotamia: Portrait of a Dead Civilization (2. Ed. Chicago 1975) S. 18-19; für die Standardisierung von Ausdrücken in Gebeten vgl. J. Goody, The Logic of Writing and the Organization of Society (Cambridge 1986) S. 38-39. 
Vereinheitlichung der Omenapodosen im 1. Jt. v.Chr. ${ }^{19}$ zur Folge hatte, erklärt werden darf, bleibt offen.

Bei den Beispielen aus den Handerhebungsgebeten beschränke ich mich auf die Darstellung von W. MAYER, Untersuchungen zur Formensprache der babylonischen «Gebetsbeschwörungen» (= Studia Pohl SM 5) (Rome 1976); die Seitenangaben beziehen sich auf seine Untersuchung. Die Stellenangaben für die physiognomischen Omina richten sich nach dem Abkürzungssystem in meiner Die babylonisch-assyrische Morphoskopie, S. 324. Zunächst werden Themen aus dem Klageteil der Gebete zitiert, in welchem der Beter sich über seine unglückliche Situation beklagt. Diesen werden die entsprechenden Parallele aus dem physiognomischen Omenwerk gegenübergestellt, d.h. die Apodosen, in welcher ebendiese unglückliche Situation dem Menschen als sein zukünftiges Schicksal prophezeit wird. Die Themen göttlicher Zuwendung und körperlichen Wohlbefindens aus dem Bitteil werden kontrastiert mit Omenapodosen, die von der Abwendung «guter Mächte» und kommendem Unwohlsein künden. Es muss betont werden, dass die zitierten Apodosen nicht nur dem physiognomischen Omenhandbuch eigen sind, sondern auch in anderen Omenkompendien bezeugt sind.

(a) Klage

- ina bīti șaltu ina sūqi puhpuhhĥu šakna/iššaknamma / iškunūnimma

«im Hause erlebe ich Streit, auf der Straße Anfeindung» (S. 109)

șalta puhpuhhâ immaršu

«Streit und Anfeindung wird ihm widerfahren», TBP 22:19

(=BaMo, S. 265)

- marsu

«krank» (S. 71)

marras, marrasat

«er/sie ist sehr krank/kränklich» Alamdimmû Tf. X:51, 53

(= BaMo, S. 120); ̌̌umma sinništu 4:146 (= BaMo, S. 160)

${ }^{19}$ S. A. L. OpPenheim, «Man and Nature in Mesopotamian Civilization», Dictionary of Scientific Biography Vol. 15 (1978) S. 642. 
- maruštu imhuranni/mahratanni

«Schlimmes ist mir widerfahren» (S. 76)

maruštu imahh̆aršu

«Schlimmes wird ihm widerfahren» KAR 395:86 (= BaMo, S. 294)

- ina kūri u nissati «in Niedergeschlagenheit und Jammer» (S. 83)

ina kūri u nissati iqatti

«in Niedergeschlagenheit und Jammer wird sie enden» Šumma sinništu 4:264 (= BaMo, S. 170)

- ìtašuš / ìtanaššuš libbi

«mein Herz ist ständig betrübt» (S. 83)

ašuštu ramānī̌šu ișsabbassu

«Trübsal wird ihn immer wieder packen» Šumma umṣātu: 10, 51 (= BaMo, S. 184, 186)

- nēmelu lā arši

«Gewinn wird er nicht bekommen» (S. 82)

nēmelšu lā irašši

«seinen Gewinn wird er nicht bekommen» šumma šE:42, 85 (= BaMo, S. 218, 222)

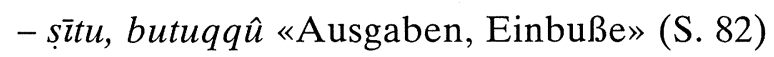

șìtu «Ausgaben» Šumma tirku:93 (= BaMo, S. 210)

butuqqûu «inbuße» Alamdimmû Tf. III:67 (= BaMo, S. 92)

- la ābu «strapazieren» (Krankheiten) (S. 92)

li ba amēla ila îb «die libu-Krankheit wird den Mann strapazieren» Alamdimmû Tf. III:132 (= BaMo, S. 96)

- lemnā hatấ idātū'a «schlimm, miserabel sind die Zeichen für mich» (S. 105) idāssa lemnet

«schlimm ist das Zeichen für sie» Šumma sinništu 4:197 sic (= BaMo, S. 164) 
- šibsāt ili

«zornige Abwendung von Gott» (S. 109)

šibsāt ili ana amēli

«Zorn des Gottes über dem Mann» ̌̌umma tirku:103 (= BaMo, S. 210)

(b) Bitte

- rišâ rēma

«hab Erbarmen mit mir» (S. 225)

ilu ana amēli rēma lā irašši

«Gott wird kein Erbramen mit dem Mann haben» Šumma ŠE:16, 77 (= BaMo, S. 214, 221)

- lamassu, ilu Bitte um Begleitung durch «gute Mächte» (S. 245f) lamassu ul irašši

«er wird keine Schutzgottheit bekommen» TBP 62:4 (= BaMo, S. 302)

ilu ul irašši

«er wird keinen Gott bekommen»TBP 22:67 (= BaMo, S. 304)

ilšu rēšišu isakkip

«sein Gott wird sein Haupt verstoßen» TBP 62:18 (= BaMo, S. 302)

- tūu libbi / šìri

«seelisches / körperliches Wohlbefinden» (S. 288)

lā țūb libbi, lā țūb š̄iri šakinšu

«kein seelisches Wohlbefinden» Alamdimmû Tf. VI 2:58, 59 (= BaMo, S. 104)

«kein körperliches Wohlbefinden» Šumma ŠE:130 (= BaMo, S. 228)

$-d \bar{\imath} n \bar{\imath} d \bar{\imath} n$

«sprich mir Recht!» (S. 221)

ina dīn ili imaqqut,

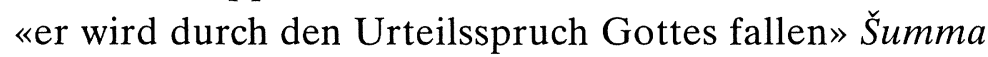
kurāru:43 (= BaMo, S. 182) 
Eine Form des Schutzes vor Unheil bildete das Amulett, welches sowohl eine Übel abwehrende Wirkung besaß als auch die Anziehung wünschenswerter Ereignisse und Güter garantieren sollte. Eine ganze Reihe von Texten mit Vorschriften zur Herstellung von Amulettsteinketten ist auf uns gekommen. Verbreitet waren u.a. Amulette, für den Fall, dass Schutzgott und Schutzgöttin eines Menschen mit ihm zürnen. In $B A M$ IV 376 Kol. i:15'$18^{\prime}$ findet sich die Beschreibung für ein derartiges Amulett mit 15 Steinen ${ }^{20}$ und der Zweck: 15 abnū ilšu u ištaršu ittī̌šu zenû. Diese Aussage findet ihr Gegenstück in einer Vorzeichendeutung aus

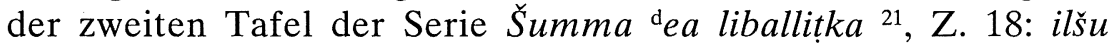
ittī̌su zeni, «sein Gott zürnt mit ihm.»

Von anderen Amuletten glaubte man, sie würden etwa Profit, Fülle und Gewinn (išdih̆u, tuḩdu und nēmelu) versprechen, wie etwa das in $B A M$ IV $375 \mathrm{Kol}$ i:29-34 verzeichnete 9-SteineAmulett ${ }^{22}$. Ebenso wie bei dem vorangehenden Beispiel bleibt es natürlich Spekulation, ob bei einer Omenapodose, die von Defiziten, Armut, Verlusten oder Mangel ${ }^{23}$ kündigte, ein derartiges Amulett getragen wurde.

Die hier vorgestellten Schutzmechanismen zeigen jedoch keine eindeutigen Berührungspunkte zu dem physiognomischen Omenwerk. Man muss daher fragen, ob die Natur der physiognomischen Omina überhaupt die Durchführung von Abwehrritualen zulässt. Wie bekannt, wurden apotropäische Rituale durchgeführt bei einem Unheil ankündigenden Ereignis oder Geschehen, das den normalen alltäglichen Ablauf als etwas Bemerkenswertes unterbricht wie im Falle von Blitzschlag oder das Verhalten bzw. Erscheinen von Tieren und Pflanzen. ${ }^{24}$ Andere Rituale wenden sich gegen Unheil, das durch Erscheinungen, die durch einen

\footnotetext{
${ }^{20}$ Es handelt sich im einzelnen um die Steine sāmtu uqnû ḩulālu muššaru pappardilû papparminu huurāṣu arqu hilibû šubû arqu šadânu șabtu haltu mūṣu lamassu sahhu.

${ }^{21}$ Für die Bearbeitung s. W. von SOden, $Z A 71$ (1981) S. 109-121.

${ }^{22} \mathrm{Zu}$ den publizierten Parallelen gehören $B A M$ IV 370 ib:1'-2'; $B A M$ IV 373 ii:24-32; $B A M$ IV 375 iv:15-18; STT II 271 iv:1-2.

${ }^{23} \mathrm{Vgl}$. etwa die Apodosen ilappin «er wird arm werden», imț̂ি šaknüšu «Mangel ist im bestimmt», ina tamtâti ittanallak «er wird mit Verlusten leben», ibissâ immar "Geldverlust wird ihm widerfahren», lupnu sadiršu «Armut ist für ihn von Dauer», lupnu ișab assu «Armut wird ihn packen»; für die Stellenangaben vgl. BaMo, S. 36.

${ }^{24}$ Vgl. S. M. MAUL, Zukunftsbewältigung, S. 115-118; 229-372.
} 
Prozess enstanden sind, angezeigt nurde; dazu gehören die sog. Opferschau-Namburbis ${ }^{25}$. Auch bestimmte menschliche Verhaltensmuster -sowohl bewussten als auch unbewussten Charaktersgalten als Unglück bringend wie etwa sexuelle Kontakte zwischen Mensch und Tier ${ }^{26}$, Nachlässigkeit in der Durchführung von Ritualen ${ }^{27}$ und Probleme bei der Wiedervereinigung getrennt lebender Ehepartner ${ }^{28}$. Allen diesen Omina ist gemein, dass sie einen punktuellen Moment erfassen, sei es in Zeit oder Raum. D.h. die Opferschau gilt für einen speziellen Moment, obgleich die Formation auf einen Prozess zurückgeht. Ähnlich sind auch Omina aus Missgestaltungen wie in der tetralogischen Omenserie Summa izbu verzeichnet zu werten: wichtig ist hier der Moment der Geburt. Andere Omina wiederum sind zeitlich an Monate gebunden. ${ }^{29}$ Schliesslich handeln auch die Omina über menschliche Aktivitäten, für die Löserituale bekannt sind, von einem bestimmten Zeitpunkt.

Hinter der Bezeichnung «physiognomisches Omen» verbergen sich Beobachtungen diverser Wertigkeit: Zum einen Beschreibungen der unterschiedlichen Formen der menschlichen Statur, die angeboren sind. Es handelt sich um Zustandsformen, die von Beständigkeit sind, denn Erwähnungen von durch Unfall, Krankheit oder Kriegsverletzung entstellten Körpergliedern fehlen im physiognomischen Omenwerk. Auf der anderen Seite finden sich Erscheinungen, die im Laufe des Lebens erworben wurden, also auf einen Prozess zurückgehen - dazu gehören etwa Gesichtsfalten und der Haarwuchs, wie sie in den Tafeln II und III des physiognomischen Omenwerkes Alamdimmû beschrieben werden. Andere Omenprotasen handeln von Charaktereigenschaften und bewussten wie auch unbewussten Verhaltensweisen, die in den Unterserien Katadimmû und Nigdimdimmû verzeichnet sind. Dazu kommt eine nicht unbeträchtliche Anzahl von Beobachtungen der Haut -insgesamt sind neun Tafeln auf uns gekommen-, d.h. Beobachtungen der Lage von Hautmerkmalen. Ähnlich den unzähligen Termini für Krankheiten scheitert die Identifizierung der

${ }^{25}$ S. S. M. MAUL, Zukunftsbewältigung, S. 432-444.

${ }^{26}$ S. S. M. MAUL, Zukunftsbewältigung, S. 415-420.

${ }^{27}$ S. S. M. MAUL, Zukunftsbewältigung, S. 421-431.

${ }^{28}$ S. S. M. MAUL, Zukunftsbewältigung, S. 409-414.

${ }^{29} \mathrm{Vgl}$. etwa das Namburbi für den Fall, dass ein Kind im Monat Nisan geboren wird; s. S. M. MAUL, Zukunftsbewältigung, S. 400-408. 
akkadischen und sumerischen Lemmata für Naevi mit unserer modernen medizinischen Nomenklatur: denn aufgrund des unterschiedlichen Krankheitskonzeptes kommen etwa den Symptombeschreibungen eine andere Wertigkeit zu. Wir können daher bislang nicht in befriedigender Weise die Termini für Hautmerkmale identifizieren, so dass offen bleibt, ob es sich um Geburtsmale oder um maligne, plötzlich auftretende Veränderungen der Haut handelt. ${ }^{30} \mathrm{Im}$ Gegensatz also zu den Unheil bringenden Vorzeichen, für die Abwehrrituale erhalten sind, stellen die physiognomischen Omina Beobachtungen eines konstanten, dauerhaften Zustandes - unabhängig ob dieser durch einen Prozess ereicht wurde- oder im Falle der Verhaltensomina Charaktereigenschaften und stetige Gewohnheiten dar. Dieser Unterschied mag die Absenz eines Abwehrmechanismus' für diesen Typus physiognomischen Omens erklären. Eine Ausnahme bilden hier die physiognomischen Omina aus Hautmerkmalen, die keine Geburtsmale sind, da sich hinter diesen Formationen verbergen können, die von einem Augenblick zum anderen eingetreten sind. D.h. dass mit diesen Omina ein bestimmter Moment erfasst wird. Vor diesem Hintergrund kommt dem Befund zweier Beschwörungen, die am Ende zweier Tafeln über Omina aus Hauterscheinungen verzeichnet sind, eine besondere Bedeutung $\mathrm{zu}$.

\section{Die BeschwÖRUNGEN IN ALAMDIMMÛ}

Zwei Tafeln des physiognomischen Handbuches führen nach den Omina eine Beschwörung an. In beiden Fällen ist der Text schlecht erhalten, so dass der unmittelbare Kontext der Beschwörung fehlt. Wir wissen daher nicht, welches Ritual -sei es die Herstellung eines Amulettes, sei es ein Apotropaicum, sei es eine Anweisung medizinisch-magischer Natur- es bei der Rezitation der Beschwörung durchzuführen galt.

Die entsprechende Zeile der Abhandlung über den «dunklen Fleck», Summa tirku 2:113 (= BaMo, S. 210) lautet:

[E]N UD HII LI KU $\mathrm{KU}_{3}[\ldots \ldots \ldots . . . .$.$] ḩe-pí HUUS TU 6$.ÉN

${ }^{30} \mathrm{~S}$. meine Ausführungen in «Hauterscheinungen in altmesopotamischer Divination und Medizin. Teil 1: Das kurāru-Mal» (in Vorbereitung). 
Vielleicht zu lesen:

[É]N u hi.li kù [ ] he-pí ḩuš TU $\mathrm{TU}_{6}$.ÉN

«[Be]schwörung: Tag der reinen Fülle [ ] zerstört ..., Wortlaut der Beschwörung.»

Bislang konnte ich die Beschwörung weder identifizieren noch Duplikate ausmachen.

Die zweite Beschwörung wird am Ende eines Textvertreters der Abhandlung über das kittabru-Hautmal zitiert; es handelt sich um die Tafel TBP 63 (= BaMo, Tf. 22-23). Der Abschnitt von insgesamt acht Zeilen (Rs. 25'-31'; die Fangzeile in Rs. 24' ausgenommen), wobei das Kolophon mindestens mit Z. 30' einsetzt, ist stark zerstört. Nur das Incipit der Beschwörung ist zitiert, die ich mit einer Beschwörung auf einem aus Sultantepe stammenden Text, der den vollen Wortlaut bietet, identifizieren konnte.

TBP 63 Rs. 25' (= BaMo, S. 228)

[ $\quad$ gu]l ki.ba gul an.ba gul.gul ki.ba gul.[

Die Beschwörung erscheint in extenso in dem Text STT II 275, Anweisungen zur Herstellung von Amulettsteinketten. Zitiert sei Kol. ii:13'-20'

13' ÉN an.ba gul ki.[ba gul]

14' an.ba gul.gul ki.[ba gul.gul]

15 ' an.ba mul [ki.ba mul]

16 ' an.ba mul.mul [ki.ba mul.mul]

17' 'lú' mul ' $x$ ' [

18' nu.zi la.ą̧ șa-'x' [

19' NENNI A NENNI sá DIN[GIR-šú $u$ ] d15-šú NENNI

$20^{\prime}$ an.ba mul.[mu]l $u$ gi.na mul $\mathrm{TU}_{6} \cdot[\mathrm{ÉN}]$

Die Beschwörung gehört zur Gruppe von im 1. Jt. v.Chr. verfassten, z.T. nicht ganz verständlichen sumerischen Beschwörungen. Falls die Lesung in Z. 18' korrekt ist, setzt mit der zweiten Hälfte der Zeile der akkadische Teil ein. Eine Übersetzung des gesamten Textes ist mir nicht möglich; aufgrund der Verbalform /gin-a/ in 20 ' gehe ich davon aus, dass es sich bei den sumerischen Verben 
um Imperative handelt ${ }^{31}$, obgleich auch eine Lesung «an ba.gul ki ba.gul, etc.» angesetzt werden könnte.

«Beschwörung: Im Himmel sei zerstört, auf der Erde sei zerstört! / Im Himmel seid zerstört, auf der Erde seid zerstört! / Am Himmel erleuchte, auf der Erde erstrahle! / Am Himmel erleuchtet, auf der Erde erstrahlet! / ... / Es erhebt sich nicht, geht! ... / NN, Sohn des NN, dessen Gott NN und dessen Göttin NN ist, / am Himmel erleuchtet und steht fest, strahle (oder Stern?). Wortlaut der Beschwörung.»

Zwar sind Zweck und Ritual der Beschwörung in STT II 275 gegeben - 10 Steine für den Fall, der Gott ist über den Menschen sehr erzürnt, welche aufgefädelt und um den Nacken des Betroffenen zu legen sind ${ }^{32}-$, doch können diese nicht ohne weiteres auf die Situation übertragen werden, die mit der Tafel von Malomina gegeben ist. Denn wie das Material medizinischer Texte zeigt, wurde oftmals dieselbe Beschwörung gegen verschiedene (Krankheits)ursachen und unter Begleitung unterschiedlicher Rituale rezitiert. ${ }^{33}$

\section{GEBET AN DEN GotT NABÛ}

Das Gebet an Nabû ist als Sultantepe-Tafel STT I 71 publiziert. W. G. LAMBERT legte eine Bearbeitung des Textes vor ${ }^{34}$, den er als «very difficult both as a piece of literature and due to the poor

\footnotetext{
${ }^{31}$ Eine weitere Beschwörung, in der Himmel und Erde sowie das Erleuchten von etwas beschrieben wird, liegt mit BAM I 28 Rs. 14'-15' vor; das Incipit lautet $14^{\prime}$ [É]N an.ta ba.mul.mul ki.ta [ba.mul.mul].

32 STT II 275 ii:10'-12': 10 abnū (NA ${ }_{4}$.MEŠ) ilu(DINGIR) amēla(NA) kám-meil $[\mathrm{x} \mathrm{x} \mathrm{x}]$ / ana pațāri $\left(\mathrm{DU}_{8}\right)$ ina turri(DUR) tašakkak(È) ÉN an.ba [gul ki.ba gul] I 7-šú 7-šú tamannüma(ŠID-nu-ma) ana kišādīšu(GÚ-š[ú tašakkan(GAR-an)]; «10 Steine: der Gott ist im Hinblick auf den Menschen sehr zornig ... um es zu lösen: auf einem Band fädelst du sie auf, die Beschwörung 'Am Himmel sei [zerstört, auf der Erde sei zerstört!]' rezitierst du sieben und siebenmal, um seinen Nacken [legst du es].»

${ }^{33}$ Für Beispiele von Beschwörungen, die weder an ein bestimmtes Ritual noch an einen bestimmten Zweck gebunden sind, s. meine Studie Die Kranken-Massage nach keilschriftlichen Quellen. Beiträge zu Medizin und Magie im Alten Orient (in Vorbereitung).

${ }^{34}$ «The Sultantepe Tablets, a Review Article», $R A 53$ (1959) S. 134-138; ein weiteres Duplikat von STT I 71 wurde von W. vON SODEN veröffentlicht, «Duplikate aus Ninive», JNES 33 (1974) S. 340-341.
} 
state of preservation and the many errors of the scribe» ${ }^{35}$ bezeichnete. Von Interesse ist hier Z. 20 des Textes:

\section{telê alamdimmê [lemn] ūti ana damiqti itūru}

«Du bist fähig, eine böse Vorzeichen tragende Gestalt in eine mit guten Omina behaftete zu kehren»

Dieser Zeile dürfen wir vielleicht entnehmen, dass mittels eines Gebetes an den Gott Nabû die schlechte «Aura» eines Menschen in das positive Gegenteil gewendet werden konnte. Wie E. Cassin ausführte ${ }^{36}$, galt der Gott Nabû im 1. Jt. v. Chr. als derjenige, der für Lahme und Debile zuständig war. Mit dem Epitheton sāniq mithurti, «der, der die Harmonie prüft», welches von einigen Ausnahmen abgesehen allein dem Gott Nabû zukam, wurde dieser Aufgabenbereich umschrieben. Im physiognomischen Omenwerk gibt es zwar keinen Hinweis darauf, daß schwere Körperbehinderungen beschrieben werden, doch zeugen zwei Stellen von körperlichen Gebrechen: In Summa sinnistu 4:229 (= BaMo, S. 166) werden die Füße einer Frau mit pussala als «abgedreht» oder «verkrümmt» bezeichnet. Das Verb ist in der Form Passalu und Pussulu auch als Personenname bezeugt ${ }^{37}$. Die zweite Stelle - eine Omenapodose, die als hapax legomenon bezeugt ist, ist in einer Tafel über das $p \bar{\imath} n d \hat{u}-$ Mal belegt (Z. 122, BaMo S. 200: uhtammar ul itarraș, «Er wird körperlich immer mehr verkümmern und nicht in Ordnung kommen».

\section{ABSCHLIEßENDE BEMERKUNG}

Wie mag der altmesopotamische Mensch mit einem durch ein physiognomisches Omen angekündigtes Unheil umgegangen sein? Die inhaltlichen Parallelen zwischen Omenapodosen und ŠuilaGebeten sowie Amuletten weisen zwar auf eine innere Verbindung hin, doch sind die Phrasen so stark standardisiert, dass sich

35 «The Sultantepe Tablets, a Review Article», S. 134.

${ }^{36}$ E. CASSIN, «Le droit et le tordu II», Le semblable et le différent, S. 76-81.

$37 \mathrm{Vgl}$. H. WAETzOLDT, «Der Umgang mit Behinderten in Mesopotamien», in Behinderung als pädagogische und politische Herausforderung, ed. M. LIEDTKE (Bad Heilbrunn 1996) S. 81; s. auch H. HolmA, Die assyrisch-babylonischen Personennamen der Form quttulu mit besonderer Berücksichtigung der Wörter für Körperfehler (Helsinki 1914) und J. J. STAMM, Die akkadische Namengebung (= $M V A e G$ 44) (Leipzig 1939) S. 264-267. 
ein konkreter Bezug auf die Physiognomie nicht ergibt. Auch lässt der Charakter des überwiegenden Teiles physiognomischer Omina Zweifel aufkommen, ob es rituelle Abwehrmechanismen gegen den Typus nicht an einen bestimmten Moment in Zeit und Raum gebundener Omina gab. Möglicherweise deutet eine Passage aus dem Text STT I 71 daraufhin, dass der Mensch durch ein Gebet sein unheilvolles Schicksal wenden konnte und dass dieses an den Gott Nabû gerichtet wurde. (In diesem Kontext sei erwähnt, dass sechs Texte zur Physiognomik aus Nabûs Tempel, dem Ezida in Nimrud, stammen: CTN $473^{38}$, CTN $474^{39}$, CTN $475^{40}$, CTN 4 $76^{41}, C T N 488^{42}$ und eine Kopie des Kataloges der Tafelanfänge

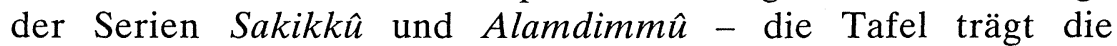
Unterschrift nișirti é.[zi.da], 'Geheimnis des Ezida' [ND 4358+ Z. A 92] ${ }^{43}$.) Im Falle von physiognomischen Omina über momentan auftretende Hautveränderungen wurde, wie die Abhandlungen Summa tirku und Summa kittabru zeigen, eine (oder mehrere?) Beschwörungen rezitiert, wobei Hinweise auf das an die Rezitation der Beschwörung gebundene Ritual noch fehlen.

\footnotetext{
209.

${ }^{38}$ Duplikat der Tafel über den «dunklen Fleck» Summa tirku, s. BaMo, S. 204-

${ }^{39}$ Duplikat der Serie von Verhaltensomina Summa Katadugĝ̂ «Wenn der Ausspruch», s. BaMo, S. 130-136.

${ }^{40}$ Text, der zu der Tafel über die Hauterscheinung umșatu gehört; s. BaMo, S. 194.

${ }^{41}$ Fragment mit Körpermal-Omina; s. BaMo, S. 320

${ }^{42}$ Text mit Verhaltensomina; s. BaMo, S. 316-317.

${ }^{43}$ Zitiert nach der Bearbeitung von I. L. FINKEL, «Adad-apla-iddina, Esagilkīn-apli, and the Series SA.GIG», S. 152.
} 


\section{RESUMEN}

En nuestro estudio Die babylonisch-assyrische Morphoskopie, Wien 2000 (= $A f O B h .27$ ), incluimos una discusión sobre el uso que pudo tener el libro cuneiforme sobre presagios fisiognómicos denominado, en acadio, Alamdimmû. Por analogía con los tratados de morfoscopia en lengua griega, latín, siríaco, árabe y hebreo, propusimos entonces que Alamdimmî pudo servir para examinar al personal antes de su posible incorporación al servicio del rey, o bien, si se trataba de mujeres, antes de que contrajesen matrimonio. En este estudio planteamos la posibilidad de que, si se producía un presagio fisiognómico ominoso, la persona implicada podía recurrir a medidas apotropaicas o de otro tipo para librarse del destino funesto que le aguardaba.

PALABRAS CLAVE: Antigua Mesopotamia, fisiognómica, destino, presagios, conjuros, oraciones.

\section{SUMMARY}

In my Die babylonisch-assyrische Morphoskopie, Wien 2000 (=AfO Bh. 27) I discussed the practical use of the cuneiform handbook of physiognomic omens, Alamdimmû. In comparison with morphoscopic treatises in Greek, Latin, Syriac, Arabic, and Hebrew I came to the conclusion that also the Akkadian handbook served as instrument for the checking and examination of people once they would enter the service of the king or, when applied to women, once they would get married. In the present paper I pose the question whether in case of an illportending physiognomy the person described in the Alamdimmû treatise could himself perform apotrapaic rituals or undertake other protective measures.

KEYWORDS: Ancient Mesopotamia, physiognomy, destiny, omens, spells, prayers. 